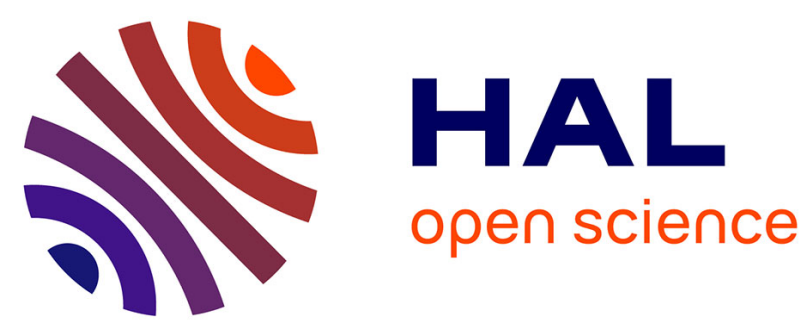

\title{
Interactions between chromosomes, microfilaments and microtubules revealed by the study of small GTPases in a big cell, the vertebrate oocyte
}

\author{
Marie-Hélène Verlhac, Julien Dumont
}

\section{- To cite this version: \\ Marie-Hélène Verlhac, Julien Dumont. Interactions between chromosomes, microfilaments and micro- tubules revealed by the study of small GTPases in a big cell, the vertebrate oocyte. Molecular and Cellular Endocrinology, 2008, 282 (1-2), pp.12. 10.1016/j.mce.2007.11.018 . hal-00531966}

\author{
HAL Id: hal-00531966 \\ https://hal.science/hal-00531966
}

Submitted on 4 Nov 2010

HAL is a multi-disciplinary open access archive for the deposit and dissemination of scientific research documents, whether they are published or not. The documents may come from teaching and research institutions in France or abroad, or from public or private research centers.
L'archive ouverte pluridisciplinaire $\mathbf{H A L}$, est destinée au dépôt et à la diffusion de documents scientifiques de niveau recherche, publiés ou non, émanant des établissements d'enseignement et de recherche français ou étrangers, des laboratoires publics ou privés. 


\section{Accepted Manuscript}

Title: Interactions between chromosomes, microfilaments and microtubules revealed by the study of small GTPases in a big cell, the vertebrate oocyte

Authors: Marie-Hélène Verlhac, Julien Dumont

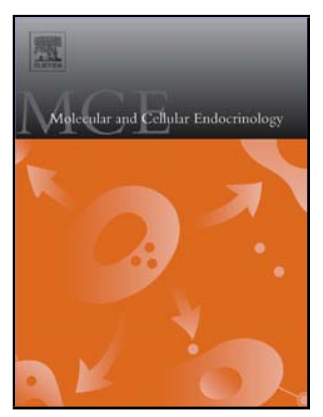

PII:

S0303-7207(07)00425-X

DOI: doi:10.1016/j.mce.2007.11.018

Reference: MCE 6752

To appear in: $\quad$ Molecular and Cellular Endocrinology

Please cite this article as: Verlhac, M.-H., Dumont, J., Interactions between chromosomes, microfilaments and microtubules revealed by the study of small GTPases in a big cell, the vertebrate oocyte, Molecular and Cellular Endocrinology (2007), doi:10.1016/j.mce.2007.11.018

This is a PDF file of an unedited manuscript that has been accepted for publication. As a service to our customers we are providing this early version of the manuscript. The manuscript will undergo copyediting, typesetting, and review of the resulting proof before it is published in its final form. Please note that during the production process errors may be discovered which could affect the content, and all legal disclaimers that apply to the journal pertain. 
Interactions between chromosomes, microfilaments and microtubules revealed by the study of small GTPases in a big cell, the vertebrate oocyte

Marie-Hélène Verlhac* and Julien Dumont ${ }^{1}$

Equipe Divisions Méiotiques chez la souris, CNRS/Université Pierre et Marie Curie,

UMR7622, Bat. C, 5e, 9 quai Saint Bernard 75005 Paris, France

${ }^{1}$ present address: Ludwig Institute for Cancer Research, Department of Cellular and

Molecular Medicine, UCSD Biomedical Sciences Graduate Program, La Jolla, CA 92093-

0653, USA

* corresponding author: verlhac@ccr.jussieu.fr

Running title: small GTPases in mouse oocytes

Key words: Meiosis, small GTPases, mouse, oocyte

Number of Characters: 22035 


\begin{abstract}
Meiotic divisions during oogenesis in higher eukaryotes are extremely asymmetric giving rise to one gamete, the oocyte, and two polar bodies. In most species, this asymmetric partitioning relies on the eccentric positioning of meiotic spindles. Recent work performed in mouse and frog oocytes has suggested the involvement of small GTPases, such as Cdc42, Rac and Ran both in the control of spindle organization and positioning. The present review summarizes these findings that shed light on the molecular mechanisms by which small GTPases control asymmetric cell divisions in vertebrate oocytes.
\end{abstract}




\section{Main Text}

Spatial and temporal control of cell division ensures equal segregation of chromosomes between two daughter cells. Female meiotic divisions give rise to daughter cells of different sizes: a big oocyte and two tiny polar bodies. In vertebrate oocytes, asymmetric meiotic divisions require both the formation of a functional spindle, which positions at the cortex, as well as the restriction of the cleavage furrow at the plasma membrane overlying the chromosomes. Spatial and temporal coordination of these processes relies on signaling properties of meiotic chromosomes, which control proper microfilaments and microtubules organization.

With recent findings in mouse and Xenopus oocytes, we will review how small GTPases control essential aspects of meiotic divisions in vertebrate oocytes.

\section{Morphological events of meiotic maturation}

Oocytes of all species are arrested in prophase I of meiosis in the ovary. Meiotic maturation then ends the process of meiosis with the succession of two asymmetric divisions. Although they occur with different kinetics in mouse and Xenopus, the successive steps are very similar. Physiologically, a hormonal surge (Luteinizing Hormone in mouse and progesterone in Xenopus) releases oocytes from the prophase I arrest. However, meiosis resumption can also be triggered in vitro by using chemical treatments, which makes it possible to follow the various steps of maturation. It begins with nuclear envelope breakdown (NEBD), also called germinal vesicle breakdown (GVBD, Figure 1). The first meiotic spindle emanates slowly from microtubule organizing centres (MTOCs) around the condensing chromosomes in the mouse or at the basal part of the nucleus in Xenopus. The migration of the spindle toward the egg cortex starts shortly after GVBD in Xenopus while it takes place only after a bipolar spindle has been assembled in the mouse (Verlhac et al., 2000). In mouse oocytes, the first meiotic spindle moves along its long axis with the pole closest to the cortex leading the way, via a microfilament-dependant process (Verlhac et al., 2000). As the chromosomes move to the close proximity of the cortex, they induce its differentiation, which results in a local accumulation of actin microfilaments and a lack of microvilli (Longo and Chen, 1985; Maro et al., 1986; Verlhac et al., 2000). This is supposed to restrict the progression of the cleavage furrow to the differentiated area overlying the chromosomes, both in meiosis I and II. By restricting the size of the polar bodies, oocytes retain most of the maternal stores for further development. After first polar body extrusion, oocyte enters the second M-phase where a 
meiotic spindle forms rapidly at the periphery of the oocyte with its long axis parallel to the cortex (Figure 1). In most vertebrate species, the cell cycle arrests in metaphase II, with a stable spindle anchored to the cortex. After fertilization, a microfilament-dependant rotation of the spindle takes place allowing its perpendicular orientation relative to the cortex, and the second polar body is extruded.

Hence, both meiotic divisions require a cortical differentiation to restrict the progression of the cleavage furrow. In addition, the asymmetry of the first division is achieved by the migration of the spindle and its anchoring to the cortex while the second division requires the cortical anchoring of the spindle and its rotation.

\section{Small GTPases}

The superfamily of small GTPases can be divided into five major subfamilies: Ras (the founding member), Rab, Arf, Rho and Ran. This review will focus only on the last two. The Rho subfamily can be subdivided into three groups: Rho, Rac and Cdc42. Each small GTPase functions as a molecular switch cycling between an active GTP-bound and inactive GDPbound forms (for review (Van Aelst and D'Souza-Schorey, 1997). There are two main classes of regulatory proteins that control this cycle: GEFs (Guanine-nucleotide Exchange Factors) promote the GTP loading of small GTPases and GAPs (GTPase Activating Proteins) stimulate the low intrinsic GTPase activity and thus their inactivation (for reviews Jaffe and Hall, 2005; Moon and Zheng, 2003; Zheng, 2001). Hence local activation/inactivation of these small GTPases can be controlled by the subcellular localization of their regulatory proteins.

Although GTPases of the Rho subfamily are involved in various processes such as regulation of gene expression or enzymatic activities and control of microtubule dynamics, their bestcharacterized function is the control of the actin cytoskeleton. The activation of Rho, Rac, or Cdc42 leads to the assembly of contractile acto-myosin filaments, actin-rich lamellipodia, and actin-rich filopodia, respectively (Hall A., 2005). Schematically, Cdc42 and Rac promote actin polymerization by activating Arp2/3, one of the major actin polymerization factors. Activation of Arp2/3 initiates a branched microfilament network. Proteins of the Rho subgroup interact with formins, another class of actin polymerization factors, leading to the assembly of straight microfilaments.

The small GTPase Ran is the only member of its family. Ran was first identified as a regulator of nucleo-cytoplasmic trafficking. Most of our understanding of the mode of action 
of the Ran GTPase comes from early studies on nuclear trafficking. Cargos, containing NLS (Nuclear Localization Signal), are imported into the nucleus through binding to import receptors, such as Importins. Once inside the nucleus, RanGTP binds to Importins thereby promoting the release of the cargos. The Importin-RanGTP complex is then exported outside the nucleus where cytosolic RanGAP inactivates RanGTP. The reverse process takes place when cargos, containing an NES (Nuclear Export Signal), bind to the export receptor, Crm1. Nuclear RanGTP binds to this complex allowing it to be transported outside the nucleus. In addition to this role, Ran also controls mitotic checkpoint, nuclear envelope assembly and spindle assembly. Ran regulates spindle assembly in a manner similar to nuclear trafficking. During M-phase, various microtubule associated proteins (MAPs) containing NLS, also called SAFs (Spindle Assembly Factors), are sequestered by Importins except in the region of the cell where RanGTP binds to Importins thereby promoting their release and activation (Zheng, 2004).

\section{Local activation of small GTPases in vertebrate oocytes}

\section{Ran GTPase}

During M-phase, microtubules are nucleated around chromosomes via the activity of the small Ran GTPase (for reviews see (Gruss and Vernos, 2004; Zheng, 2004). The RanGEF, RCC1, is concentrated on chromosomes when RanGAP is cytosolic. Since opposing activities are spatially segregated, a gradient of RanGTP is produced both in Xenopus extracts and in mitotic cells, with high RanGTP near the chromatin and low RanGTP concentrations away from the chromosomes (Caudron et al., 2005; Kalab et al., 2006; Kalab et al., 2002).

Direct evidence for a RanGTP gradient in mouse oocytes has been recently demonstrated using a FRET-based probe (Dumont et al., 2007b). As expected from studies in other systems, the RanGTP level peaks in the vicinity of chromosomes whereas it declines linearly away from the chromatin area, creating a broad gradient all over the oocyte (Figure 1). This local RanGTP accumulation accompanies chromosomes migration toward the cortex suggesting that it might provide a spatial clue of their position within the cell (Dumont et al., 2007b).

\section{Rho GTPases}

Using a fluorescent probe that binds specifically to RacGTP, Halet and Carroll (2007) have elegantly showed the localization of active Rac in mouse oocyte. Prior to spindle migration in immature oocytes, RacGTP is uniformly enriched all over the cortex. As the spindle migrates 
toward the cell cortex, this activity becomes restricted to the region overlying the spindle, where actin filaments accumulate, and then to the cortex of the first polar body. After the first division, active Rac accumulates in the cortical region overlying the MII chromosomes independently of the presence of microtubules (Halet and Carroll, 2007). This observation shows that meiotic chromosomes emit a signal that triggers RacGTP accumulation in their vicinity. Since this accumulation is an "at distance" effect that does not require any direct contact of chromosomes with the cortex, it is tempting to speculate that a gradient of active RacGEF is centered on meiotic chromosomes and leads to the activation of Rac in their vicinity. Yet, actin accumulation at the cortex after injection of a dominant negative Rac, suggests that this local activation of Rac does not participate in the mechanism of cortical polarisation (Halet and Carroll, 2007). Therefore in mouse oocytes, the position of the chromosomes is translated into a RanGTP gradient while their proximity to the cortex induces a local RacGTP accumulation. Future work will examine wether a direct relationship exists between the RanGTP gradient and the local activation of RacGTP at the cortex. An interesting experiment would be to analyze RacGTP activation in a context where the RanGTP gradient is disrupted.

Interestingly, in Xenopus oocytes, active $\mathrm{Cdc} 42$ and RhoA concentrate in the region overlying the meiotic spindle in MI, similarly to RacGTP accumulation in mouse oocytes (Ma et al., 2006). However, it has been hypothesized that this local accumulation of RhoA and Cdc42 requires spindle microtubules (Ma et al., 2006). It would be interesting to test whether chromosomes alone are able to induce this cortical activation of RhoA and Cdc42, as in the case of Rac in mouse oocytes. Another important experiment would be, using a similar approach, to follow the localisation of active Rac, $\mathrm{Cdc} 42$ and RhoA during first meiotic spindle migration in order to determine potential regions of overlapping activities of these GTPases in the cortex of mouse oocytes.

\section{Function of small GTPases in spindle formation and positioning}

In most cells, spindle assembly involves the concerted action of two mechanisms: "Searchand-capture" and "local assembly". Search-and-capture relies on centrosomes activity, and induces assembly of microtubules from spindle poles. Local assembly relies on the small Ran GTPase, which leads to assembly of microtubules from chromosomes that are progressively sorted and organized in a bipolar array by molecular motor proteins. Mouse and Xenopus 
oocytes are devoid of centrosomes, thus it has been hypothesized that in these models meiotic spindle assemble via the local assembly mechanism only (Gard et al., 1995; Huchon et al., 1981; Szöllösi et al., 1972).

Unexpectedly, a meiosis I spindle, which is able to segregate homologous chromosomes, forms both in mouse and Xenopus oocytes, even in conditions where the RanGTP gradient no longer exists (either in a context of high RanGTP levels or low RanGTP levels all over the cytoplasm; (Dumont et al., 2007b). Moreover the analysis of the role of the RanGTPase during spindle formation in vertebrate oocytes has revealed a new property of the two meiotic divisions: spindle formation in meiosis II is much more sensitive to alterations of the RanGTP gradient than that of the meiosis I (Table 1). This could be related to the fact that spindle formation in meiosis I is slower than in meiosis II. Indeed, it has been proposed that in rapidly dividing cells containing centrosomes, the local accumulation of RanGTP and the subsequent microtubule nucleation close to chromosomes introduce a bias, which improves the efficiency of the search-and-capture mechanism allowing faster spindle formation (Wollman et al., 2005).

In vertebrate oocytes, the first meiotic spindle forms around chromosomes in the region of the cell where the nucleus was located at the time of GVBD. In addition, during meiosis I, vertebrate oocytes must coordinate the morphogenesis of the spindle and its positioning within the cell. Mouse and Xenopus meiotic spindles are organized from acentriolar MTOCs and are thus anastral (Huchon et al., 1981; Szöllösi et al., 1972). Therefore, in contrast to many cell types, spindle positioning does not involve interactions between astral microtubules and actin filaments of the cortex. In mouse oocyte, nocodazole-induced depolymerization of microtubules does not prevent chromosomes movement to the cortex suggesting that they play a minor role, if any, in spindle migration (Verlhac et al., 2000). In contrast, cytochalasinD treatment completely inhibits spindle movements, which is similar to the phenotype observed in the absence of Formin-2, a straight actin filament nucleator. These results suggest that, in mouse oocyte, MI spindle migration is under the control of actin filaments nucleated by Formin-2 (Dumont et al., 2007a; Leader et al., 2002; Verlhac et al., 2000). To date, actin filaments connecting chromosomes to the cortex in meiosis I have never been visualized directly, suggesting that these actin filaments are very thin, probably not organised like the cables observed in yeast and migrating cells. In Xenopus oocytes, the role of microtubules in chromosomes movement is unclear. On the other hand, microfilaments and the unconventional Myosin X are clearly involved in this process (Weber et al., 2004). 
Nonetheless, in both experimental models, interactions between actin and spindle microtubules are essential for the asymmetry of the divisions. Thus, perturbing the function of Rho GTPases should lead to spindle positioning defects. Unexpectedly, the use of dominant negative Rac or Cdc42 on mouse maturing oocytes, induces elongation of MI spindles which fail to efficiently segregate homologous chromosomes (Halet and Carroll, 2007; Na and Zernicka-Goetz, 2006). Dominant negative Rac induces spindle elongation as well as chromosome dispersion on the spindle (Halet and Carroll, 2007), whereas dominant negative Cdc42 only induces spindle elongation (Na and Zernicka-Goetz, 2006), suggesting that these two molecules have different unknown targets (Table 1). In mitotic cells, the use of dominant negative $\mathrm{Cdc} 42$ has suggested that it regulates kinetochore microtubule attachment, but not spindle dynamics, leading to cell cycle defects (Yasuda et al., 2004). However, this role has not been confirmed in mitotic cdc42-/- MEFs or ES cells, which harbor normal cell cycle progression (for an extensive review see Wang and Zheng, 2007). Moreover, Cdc42 and Rac are known to indirectly inhibit a microtubule-destabilizing factor of the Op18/stathmin family (Daub et al., 2001). Op18/stathmin is a catastrophe-promoting factor that interacts both with microtubule plus ends to promote microtubules disassembly and with tubulin dimers to inhibit polymerization (Cassimeris, 2002). Thus inhibiting Rac and Cdc42 should induce spindle shortening rather than elongation. It is also difficult to understand how active Rac, which concentrates in the cortex overlying the chromosomes during meiosis I spindle migration, can modulate spindle morphogenesis "at distance" (Halet and Carroll, 2007). One possibility is that a fraction of RacGTP, below the detection level of the probe used by Halet and Carroll, is localized elsewhere in the oocyte. Alternatively, Rac substrates activated in the cortex could be transferred to the spindle where they regulate spindle morphogenesis. In mouse oocytes, the first meiotic spindle forms as it migrates, so one could imagine that perturbing the function of these Rho GTPases would affect microfilaments (their dynamics or anchoring) supporting spindle migration, which in turn would induce inappropriate tension on the MI spindle indirectly creating elongation. This could explain why dominant negative Rac or Cdc42 have no effect on the meiotic spindle in Xenopus oocytes, where the role of actin filaments in spindle positioning has not been demonstrated (Ma et al., 2006). Eventually it would be important to know the expression level of dominant negative Rac or Cdc42 over the endogenous proteins to estimate whether they could induce non-specific inhibition/activation of proteins controlling spindle dynamics such as the Ran GTPase (Halet and Carroll, 2007; Na and Zernicka-Goetz, 2006). 


\section{Function of small GTPases in cortical differentiation}

As mentioned previously, chromosomes induce cortical differentiation through an activity that acts "at distance". In Xenopus oocytes, the use of a dominant negative Cdc42 inhibits first polar body extrusion. This phenotype results from abnormally low cortical actin accumulation above the meiosis I spindle. Thus in this model, $\mathrm{Cdc} 42$ seems to be required for the cortical polarization that occurs prior to polar body extrusion (Ma et al., 2006). It would be interesting to know if this is also the case in mouse oocytes. However, the spindle elongation observed after Cdc42 inhibition also prevents proper spindle positioning (probably by mechanical constraints, each pole of the spindle reaching one side of the cortex) and thus it does not allow to directly test this hypothesis. Using cytoplast of MII mouse oocytes, Deng et al. (Deng et al., 2007) showed that DNA-coated beads mimic the local accumulation of actin and phosphorylated myosin II normally induced by chromosomes. They further show that the activity associated with DNA-coated beads acts in a dose and distance-dependent manner. Eventually they demonstrate that RanGTP is required for chromatin-induced cortical differentiation. Indeed, cytoplasts containing DNA-coated beads co-injected with inactive Ran (RanT24N mutant) are unable to induce this differentiation, even though they are able to induce spindle formation. Since this cortical differentiation is required to restrict the progression of the cleavage furrow and thus the size of the polar bodies, these results are consistent with the observation that RanT24N expressing MII oocytes, when subjected to Strontium-activation, cleave symetrically instead of extruding a second polar body (Dumont et al., 2007b). One possibility is that RanGTP is essential to maintain MII spindle anchoring to the cortex. Alternatively, the differentiated area, which overlies the chromosomes, could be essential to restrict cleavage furrow progression but non-essential for cleavage furrow initiation per se. Eventually, during meiosis II, increasing or decreasing RanGTP levels leads to similar effects on meiosis II spindles as well as on cortical differentiation showing that in MII, the presence of a RanGTP gradient is essential for maintaining both a normal bipolar spindle organization and its above cortical differentiated area (Deng et al., 2007; Dumont et al., 2007b). The overexpression of inactive Rac in MII arrested oocytes prevents spindle anchoring and second polar body extrusion but not MII spindle organization once it has been formed (Halet and Carroll, 2007). Based on the effect of overexpression of the inactive Rac in MI and MII, one could speculate that Rac regulates anchoring of the meiotic spindles to the 
cortex, but not the formation of the actin cap per se, which would depend on the presence of a RanGTP gradient.

\section{Conclusions}

The study of small GTPases in vertebrate oocytes has unraveled new unexpected roles for these proteins. The RanGTPase would regulate mostly meiosis II spindle formation but also actin filaments involved in cortical differentiation whereas Rac and Cdc42 have been shown to regulate mainly meiosis I spindle formation. These studies on the role of small GTPases in vertebrate oocytes have been performed mainly using dominant-negative or constitutively active mutants hence until genetic approaches confirm these functions, we must still be cautious. Nevertheless, new targets of these small GTPases are yet to be identified to answer challenging questions to come. Are there any specific targets of Ran which could explain why in vertebrate oocytes, meiosis II can be compromised to a much greater extent than meiosis I by RanGTP gradient alterations? What are the RanGTP targets involved in cortical actin organization? Does their binding to Importins also regulate them? Does RhoA have a role in controlling Formin-2-dependant spindle migration in mouse oocytes? What are the targets of Rac and Cdc42 that induce meiosis I spindle elongation? Are these targets actin and/or microtubule regulators? 


\section{Acknowledgements}

We thank Karen W. Lee for critical reading of this manuscript. This work was supported by grants from the Association pour la Recherche sur le Cancer (ARC3877 to M.H.V.) and from the Agence Nationale pour la Recherche (ANRNT05-1-43120 to M.H.V.). JD was supported by an ARC (Association pour la Recherche contre le Cancer) doctoral fellowship. 


\section{References}

Cassimeris, L. (2002). The oncoprotein 18/stathmin family of microtubule destabilizers. Current Opinion in Cell Biology 14, 18-24.

Caudron, M., Bunt, G., Bastiaens, P. and Karsenti, E. (2005). Spatial coordination of spindle assembly by chromosome-mediated signaling gradients. Science 309, 1373-1376.

Daub, H., Gevaert, K., Vandekerchhove, J., Sobel, A. and Hall, A. (2001). Rac/Cdc42 and p65PAK Regulate the Microtubule-destabilizing Protein Stathmin through Phosphorylation at Serine 16. The Journal of Biological Chemistry 276, 1677-1680.

Deng, M., Suraneni, P., Schultz, R. M. and Li, R. (2007). The RanGTPase mediates chromatin signaling to control cortical polarity during polar body extrusion in mouse oocytes. Developmental Cell 12, 301-308.

Dumont, J., Million, K., Sunderland, K., Rassinier, P., Hyunjung, L., Leader, B. and Verlhac, M.-H. (2007a). Formin-2 is required for spindle migration and for late steps of cytokinesis in mouse oocytes. Developmental Biology 301, 254-265.

Dumont, J., Petri, S., Pellegrin, F., Terret, M.-E., Bohnsack, M. T., Rassinier, P., Georget, V., Kalab, P., Gruss, O. J. and Verlhac, M.-H. (2007b). A centriole-and RanGTPindependent spindle assembly pathway in meiosis I of vertebrate oocytes. The Journal of Cell Biology 176, 295-305.

Gard, D. L., Affleck, D. and Error, B. M. (1995). Microtubule organization, acetylation and nucleation in Xenopus laevis oocytes: II. A developmentam transition in microtubule organization during early diplotene. Developmental Biology 168, 189-201.

Gruss, O. J. and Vernos, I. (2004). The mechanism of spindle assembly: funcions of Ran and its target TPX2. The Journal of Cell Biology 166, 949-955.

Halet, G. and Carroll, J. (2007). Rac activity is polarized and regulates meiotic spindle stability and anchoring in mammalian oocytes. Developmental Cell 12, 309-317.

Hall A. (2005). Rho GTPases and the control of cell behaviour. Biochemical Society Transactions 33, 891-895.

Huchon, D., Crozet, N., Cantenot, N. and Ozon, R. (1981). Germinal vesicle breakdown in the Xenopus laevis oocyte: description of a transient microtubular structure. Reproduction, Nutrition, Development 21, 135-148.

Jaffe, A. B. and Hall, A. (2005). RhoGTPases: biochemistry and Biology. Annual Review of Cell and Developmental Biology 21, 201-229.

Kalab, P., Pralle, A., Isacoff, E. Y., Heald, R. and Weis, K. (2006). Analysis of a RanGTPregulated gradient in mitotic somatic cells. Nature 440, 697-701. 
Kalab, P., Weis, K. and Heald, R. (2002). Visualization of a Ran-GTP gradient in interphase and mitotic Xenopus egg extracts. Science 295, 2452-2456.

Leader, B., Lim, H., Carabatsos, M. J., Harrington, A., Ecsedy, J., Pellman, D., Maas, R. and Leder, P. (2002). Formin-2, polyploidy, hypofertility and positioning of the meiotic spindle in mouse oocytes. Nature Cell Biology 4, 921-929.

Longo, F. J. and Chen, D.-Y. (1985). Development of cortical polarity in mouse eggs: Involvement of the meiotic apparatus. Developmental Biology 107, 382-394.

Ma, C., Benink, H. A., Cheng, D., Montplaisir, V., Wang, L., Xi, Y., Zheng, P.-P., Bement, W. M. and Liu, X. J. (2006). Cdc42 activation couples spindle positioning to first polar body formation in oocyte maturation. Current Biology 16, 214-220.

Maro, B., Johnson, M. H., Webb, M. and Flach, G. (1986). Mechanism of polar body formation in the mouse oocyte: an interaction between the chromosomes, the cytoskeleton and the plasma membrane. Journal of Embryology and experimental Morphology 92, 11-32.

Moon, S. Y. and Zheng, Y. (2003). Rho GTPase-activating proteins in cell regulation. Trends Cell Biol 13, 13-22.

Na, J. and Zernicka-Goetz, M. (2006). Asymmetric positioning and organization of the meiotic spindle of mouse oocytes requires CDC42 function. Current Biology 16, 1249-1254.

Szöllösi, D., Calarco, P. and Donahue, R. P. (1972). Absence of centrioles in the first and second meiotic spindles of mouse oocytes. Journal of Cell Science 11, 521-541.

Van Aelst, L. and D'Souza-Schorey, C. (1997). Rho GTPases and signaling networks. Genes and Development 11, 2295-2322.

Verlhac, M.-H., Lefebvre, C., Guillaud, P., Rassinier, P. and Maro, B. (2000). Asymmetric division in mouse oocytes: with or without Mos. Current Biology 10, 1303-1306. Wang, L. and Zheng, Y. (2007). Cell type-specific functions of Rho GTPases revealed by gene targeting in mice. Trends Cell Biol 17, 58-64.

Weber, K. L., Sokac, A. M., Berg, J. S., Cheney, R. E. and Bement, W. M. (2004). A microtubule-binding myosin required for nuclear anchoring and spindle assembly. Nature 431, 325-329.

Wollman, R., Cytrynbaum, E. N., Jones, J. T., Meyer, T., Scholey, J. M. and Mogilner, A. (2005). Efficient chromosome capture requires a bias in the "search and capture" process during mitotic-spindle assembly. Current Biology 15, 828-832.

Yasuda, S., Oceguera-Yanez, F., Kato, T., Okamoto, M., Yonemura, S., Terada, Y., Ishizaki, T. and Narumiya, S. (2004). Cdc42 and mDia3 regulate microtubule attachment to kinetochores. Nature 428, 767-771. 
Zheng, Y. (2001). Dbl family guanine nucleotide exchange factors. Trends in Biochemical Sciences 26, 724-732.

Zheng, Y. (2004). G protein control of microtubule assembly. Annual Review of Cell and Developmental Biology 20, 867-894. 


\section{Figure Legend}

\section{Figure 1}

Schematic of meiotic maturation in mouse oocytes showing the localization of active RacGTP and of the RanGTP gradient.

GV: Germinal Vesicle; GVBD: Germinal Vesicle BreakDown; MI: metaphase I; MII: metaphase II; PB1: first polar body; CSF: Cytostatic Factor arrest in MII

\section{Figure 2}

Schematic of first polar body extrusion in Xenopus oocytes showing the localizations of active Cdc42 and RhoA

\section{Table 1}

Roles of small GTPases in the control of meiotic maturation in Xenopus and mouse oocytes 


\begin{tabular}{|c|c|c|}
\hline & Хепориs & Mouse \\
\hline Ran & $\begin{array}{l}\text { Meiotic spindle assembly } \\
\text { (Dumont et al., 2007b) }\end{array}$ & $\begin{array}{l}\text { Meiotic spindle assembly } \\
\text { Cortical polarization } \\
\text { (Dumont et al., 2007b) }\end{array}$ \\
\hline $\mathrm{Cdc} 42$ & $\begin{array}{l}\text { Fisrt polar body extrusion } \\
\text { (Ma et al., 2006) }\end{array}$ & $\begin{array}{c}\text { MI spindle assembly and } \\
\text { migration } \\
\text { Completion of meiosis I } \\
\text { (Na and Zernicka-Goetz, 2006) }\end{array}$ \\
\hline RhoA & $\begin{array}{c}\text { MI spindle migration and/or } \\
\text { anchorage } \\
\text { (Ma et al., 2006) }\end{array}$ & \\
\hline Rac & $\begin{array}{c}\text { Not invoved in migration, nor } \\
\text { anchoring nor PB } \\
\text { extrusion } \\
\text { (Ma et al., 2006) }\end{array}$ & $\begin{array}{c}\text { MI spindle assembly } \\
\text { Completion of meiosis I } \\
\text { MII spindle anchorage } \\
\text { Second polar body extrusion } \\
\text { (Halet and Carroll, 2007) }\end{array}$ \\
\hline
\end{tabular}




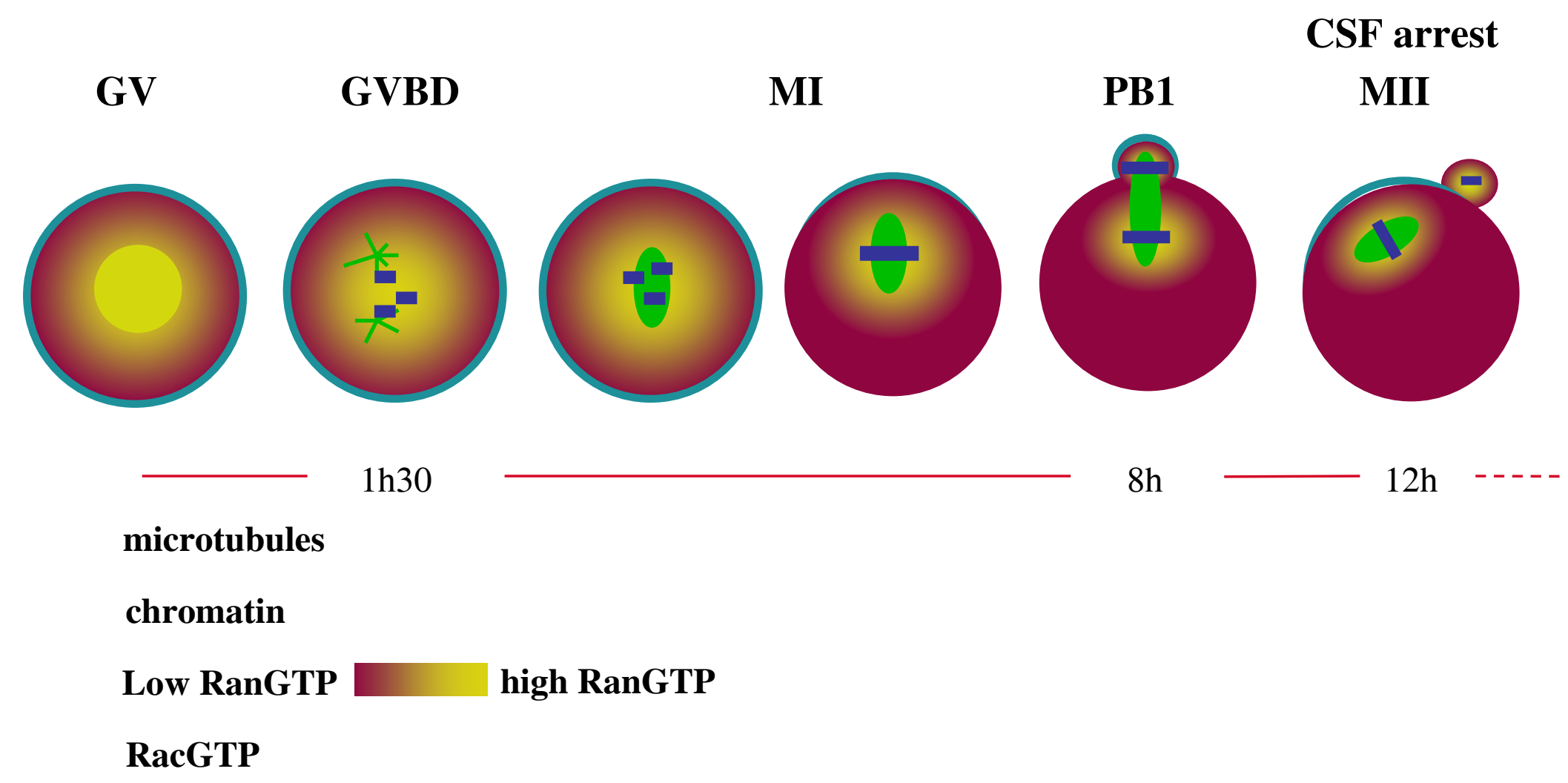

Figure 1 


$$
\text { MI }
$$

PB1

microtubules

chromatin

Cdc42-GTP

RhoA-GTP

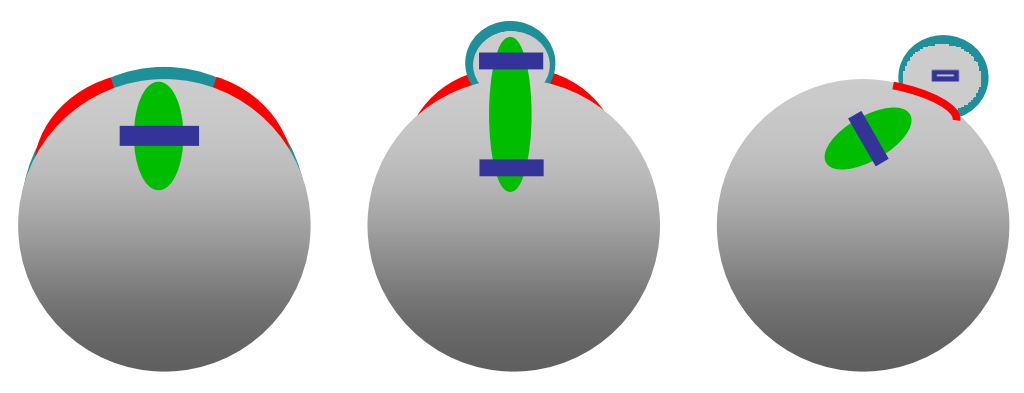

Figure 2 


\begin{tabular}{|c|c|c|}
\hline & Xenopus & Mouse \\
\hline Ran & $\begin{array}{l}\text { Meiotic spindle assembly } \\
\text { (Dumont et al., 2007b) }\end{array}$ & $\begin{array}{l}\text { Meiotic spindle assembly } \\
\text { Cortical polarization } \\
\text { (Dumont et al., 2007b) }\end{array}$ \\
\hline $\mathrm{Cdc} 42$ & $\begin{array}{l}\text { Fisrt polar body extrusion } \\
\qquad \text { (Ma et al., 2006) }\end{array}$ & $\begin{array}{l}\text { MI spindle assembly and migration } \\
\text { Completion of meiosis I } \\
\text { (Na and Zernicka-Goetz, 2006) }\end{array}$ \\
\hline RhoA & $\begin{array}{l}\text { MI spindle migration and/or anchorage } \\
\qquad \text { (Ma et al., 2006) }\end{array}$ & $?$ \\
\hline $\mathrm{Rac}$ & $\begin{array}{c}\text { Not invoved in migration,nor anchoring nor } \\
\text { PB extrusion } \\
\text { (Ma et al., 2006) }\end{array}$ & $\begin{array}{l}\text { MI spindle assembly } \\
\text { Completion of meiosis I } \\
\text { MII spindle anchorage } \\
\text { Second polar body extrusion } \\
\text { (Halet and Carroll, 2007) }\end{array}$ \\
\hline
\end{tabular}

